\title{
A review of the book Quadratic Diophantine Equations
}

\author{
by Titu Andreescu and Dorin Andrica
}

After two books on Diophantine equations, namely:

- T. Andreescu, D. Andrica, An introduction to Diophantine Equations, Gill Publishing House, Zalau (2002) and

- T. Andreescu, D. Andrica, I. Cucurezeanu, An introduction to Diophantine Equations, Birkhauser, Boston (2010)

and many other articles on this topic, professors Titu Andreescu (School of Natural Sciences and Mathematics, University of Texas at Dallas, USA) and Dorin Andrica (Faculty of Mathematics and Computer Science, "BabeşBolyai" University, Cluj-Napoca, Romania) delight readers again with Quadratic Diophantine Equations, Springer (2015), a true monograph on this important area of Number Theory.

The first chapter of the book (entitled "Why Quadratic Diophantine Equations?") is one of preliminaries, a kind background for the next chapters. The authors recall Thue's theorem, Hilbert's tenth problem, Hecke operators, Hecke groups, continued fractions, computing self-intersections of closed geodesics, standard homogeneous Eisenstein manifolds and Diophantine equations, etc.

The second chapter (entitled "Continued Fractions, Diophantine Approximation, and Quadratic Rings") introduces the readers in the theory of continued fractions, theory used in the later chapters, in order to find the minimal solution of a Pell's equation and not only. The chapter ends with a paragraph about units and norms in quadratic rings.

After these two chapters, the readers have all the theoretical support necessary to solve any type of Pell's equations or Diophantine equations reducible by Pell's equations. 
The third chapter starts with the positive Pell's equation (the authors present three methods of finding the general solution of a positive Pell's equation) and continues with the Diophantine equation $a x^{2}-b y^{2}=1$ as well as with the negative Pell's equation. This chapter ends with the presentation of results about Pell's constant and of a conjecture of Peter Stevenhagen involving this constant.

In the fourth chapter the authors discuss the general Pell's equation. There are five methods for solving the general Pell's equation. The authors present here three of these five methods: the Lagrange-Matthews-Mollin (LMM) method, the method of "brute-force search" and the method using quadratic rings. The same chapter features the equations $a x^{2}-b y^{2}=c, a x^{2}+b x y+c y^{2}=$ $N$, Thue's theorem and the equations $x^{2}-D y^{2}= \pm N$.

The fifth chapter deals with some Diophantine equations, whose solutions are reducible to Pell's type equations.

Other interesting Diophantine equations, reducible to Pell's equations are introduced in the sixth chapter. An interesting fact is that some of these equations have solutions represented in terms of the Fibonacci numbers, the Lucas numbers or the Pell numbers.

In the last chapter there are Diophantine equations involving triangular numbers, polygonal numbers, etc.

Given the wide range of Diophantine equations presented, some solved elementary and many others with approaches that involve elements of the algebraic number theory or of analytic number theory, as well as the extensive list of reference titles provided, I think that this book is a landmark in the specialty literature.

Diana Savin

"Ovidius" University, Constanţa, Romania 